\title{
QUALITY CONTROL TESTS IN SOME DIAGNOSTIC X-RAY UNITS IN BANGLADESH
}

\author{
M. Begum ${ }^{1}$, A. S. Mollah ${ }^{2}$, M. A. $\operatorname{Zaman}^{3}$ and A. K. M. M. Rahman ${ }^{4}$ \\ ${ }^{1}$ Health Physics and Radioactive Waste Management Unit, INST, AERE, \\ Bangladesh Atomic Energy Commission, Savar \\ ${ }^{2}$ Bangladesh Atomic Energy Commission, Agargaon, Dhaka, Bangladesh \\ ${ }^{3}$ Physics Department, Jahangirnagar University, Savar, Dhaka, Bangladesh \\ ${ }^{4}$ Centre for Nuclear Medicine and Ultrasound, DMCH, Bangladesh Atomic Energy Commission \\ email: go2munmun@yahoo.com, mollah_as@yahoo.com
}

\begin{abstract}
Regular implementation of quality control in diagnostic x-ray facilities is essential to provide good quality images which lead to proper diagnoses with minimum hazard and distress. Important performance tests in diagnostic radiology in Bangladesh are carried out according to a quality control protocol and the measured parameter values are compared to the relevant acceptance limits. In this work, beam alignment, field congruence, nominal focal spot, film-screen contact and half value layer for diagnostic x-ray facilities are measured by using beam alignment test tool, RMI/Victoreen collimator test tool, Bar pattern focal spot test tool, film/screen contact test tool (RMI143D), Gammex RMI step wedge and densitometer from forty different diagnostic x-ray facilities in Bangladesh. For congruence between optical and radiation fields, $77.5 \%$ are found to be within limit and $60 \%$ of facilities are within the beam alignment limit. For most of the installations, $92.5 \%$ nominal focal spot size of diagnostic x-ray machines is matched perfectly with the rating of focal spot size. In an effort to improve image quality, this study has checked the film-screen contacts of multiple facilities and found $65 \%$ to have the expected uniformity. While investigating half value layers (HVL), a measure of x-ray beam quality, it is found that none of the diagnostic X-ray installations can achieve the recommended levels.
\end{abstract}

Key words: Quality control, Beam alignment, Field congruence, Half value layer (HVL), Nominal focal spot and Acceptance limit

\section{INTRODUCTION}

Quality control in diagnostic radiology is essential to ensuring accurate diagnostic information at optimal radiation doses [1-4], thereby making it possible to reduce unnecessary radiation hazard to patients, workers and the public. In Bangladesh, many x-ray machines are installed and commissioned, ignoring radiation protection aspects and safety consideration, and are operated without a proper quality control programme. This results in contributing to a large radiation dose to human beings and also affects diagnostic image quality which may not provide accurate diagnostic information. Though $\mathrm{x}$ rays are extensively used in the diagnosis of diseases and injury all over the world improper use of $\mathrm{x}$ rays can produce biological damage because of its ionizing nature. As a result, it has long been appreciated that the irradiation of the patient should be kept to the lowest limit consistent with sufficient image quality [5-10]. Proper assessment of any disease or fracture depends on the quality of the diagnostic images [11], which are affected by many factors such as beam alignment, film-screen contact etc. Fault in any single factor may impact the final image quality because the factors are largely interdependent. Therefore, quality control in diagnostic $\mathrm{x}$-ray facilities is required for the safety and improved performance of the systems. Certain essential quality control tests, which have greater effect on the final diagnostic image quality, are investigated in the present study. Tests are conducted on some diagnostic x-ray facilities according to a quality control protocol, and the measured parameter values are compared to the relevant acceptance limits [12].

The congruence between optical and radiation fields to prevent irradiation of unwanted areas during radiography and to limit the amount of scattered radiation reaching the area of clinical interest is 
investigated. Beam alignment tests are performed to check the perpendicularity of the x-ray beam to the image receptor.

The focal spot size for diagnostic x-ray machines is checked to ensure that focal spot size and shape are not altered as a result of bombardment of electrons on the target. A small focal spot yields more detail in a radiograph and produces $\mathrm{x}$-ray images with minimum blur.

Poor contact between film and intensifying screen causes loss of resolution in the image, which occurs due to light diffusion. In this case, blur or dark images are produced within the area of poor contact. This can lead to improper diagnosis, requiring further diagnostic attempts.

In diagnostic radiology, the half-value layer (HVL) of the x-ray beam is needed in order to judge the beam quality of the $\mathrm{x}$-rays spectra.

\section{METHODS AND MATERIALS}

In the present study, different parameters essential to improve diagnostic image quality, and thus to reduce the risk of hazardous effects of ionizing $\mathrm{x}$ radiation, are investigated from forty different diagnostic x-ray facilities. These facilities are shown by code numbers. The measurement procedures followed are described below.

\subsection{Beam Alignment}

Perpendicularity of the $\mathrm{x}$-ray beam to the image receptor is measured to reduce diagnostic image distortion [12]. In case a grid is used, the distortion may be magnified and this can result in the complete loss of minute details. In the present work, the collimator test tool is placed on the film-cassette. The beam alignment tool is also kept at the center of the collimator test tool. The x-ray tube is directed over the collimator test tool at a distance of $100 \mathrm{~cm}$ from the film cassette and optical field is collimated at the marked rectangle of collimator test tool. Then film is exposed and developed. A radiograph of the beam alignment test is shown in Fig.1.

In this case, if the image of the top ball (larger shadow) intercepts the first circle, the beam is about $1.5^{\circ}$ away from the perpendicular and, if the image of the top ball intercepts the second circle, the misalignment is $3^{0}$ with a tolerance limit of about $1.5^{0}$ from the perpendicular [12]. Therefore, the performance of the x-ray unit is considered satisfactory only if the image of top ball intercepts the first circle.

\subsection{Field Congruence}

For this study, an RMI/ Victoreen collimator test tool is placed on the film cassette, and the optical field is adjusted to match with the rectangular outline of the test tool (four metal

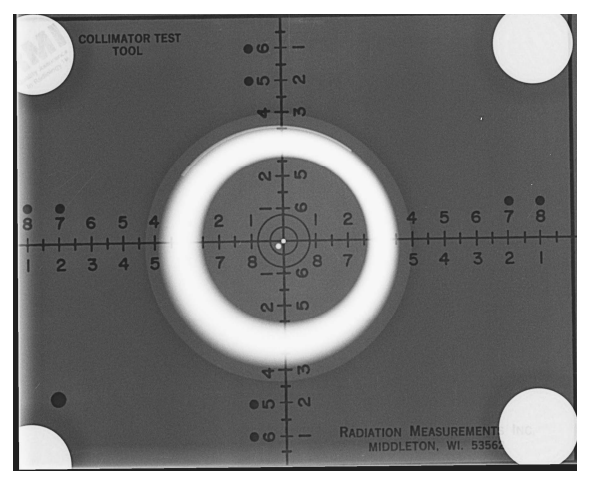

Fig.1: Radiograph of beam alignment

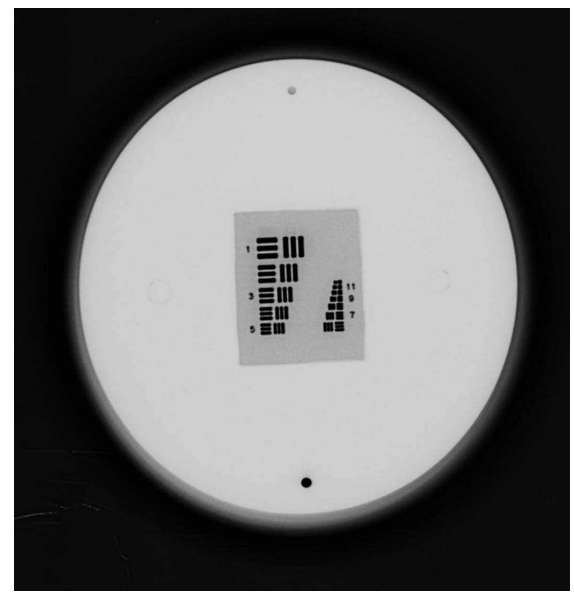

Fig.2: Radiograph of bar pattern focal spot markers are kept at the four corners of the light field). The focus-to-film distance is set at $100 \mathrm{~cm}$ before the exposure; the film is processed and the displacement error is determined from the scale appearing on the processed film. In this case, the tolerance limit of each side is $2 \%$ of FFD (Film to Focus Distance). $[12,13,14,15]$. 


\subsection{Nominal Focal Spot}

Periodic evaluation of nominal focal spot size is one of the important parameters of quality assurance in diagnostic radiology. Evaluation of nominal focal spot size is performed by placing the bar pattern focal spot test tool on the cassette containing the film. In this arrangement, the bar pattern is supported by a Perspex cylinder approximately $15 \mathrm{~cm}$ above the film-cassette. The x-ray tube is positioned over the test tool so that the x-ray target is about $45 \mathrm{~cm}$ from the bar pattern. Implied radiograph of the bar pattern focal spot test is shown in Fig. 2; groups of resolvable bars are evaluated from the processed radiographs. Nominal focal spots of the x-ray tubes are determined from the chart, given in Table 1, which relates resolution with rated focal-spot size.

\subsection{Film-Screen Contact}

Film-Screen Contact is checked by the radiographic film/ screen contact test tool (RMI 143D) in different diagnostic x-ray facilities to assess the proper contact between film and screen. For the measurement, the cassette whose film-screen contact is to be checked is loaded and placed on the x-ray machine, $100 \mathrm{~cm}$ from the tube-target.

Table 1: Nominal focal spot size from bar pattern test tool

\begin{tabular}{|c|c|c|c|c|}
\hline Group & $\mathrm{lp} / \mathrm{mm}$ & $\begin{array}{c}\text { Diameter }(\mathrm{mm}) \text { in plane of } \\
\text { focal spot }\end{array}$ & $\begin{array}{c}\text { Focal spot } \\
\text { (Nominal) } \\
(\mathrm{mm})\end{array}$ & $\begin{array}{c}\text { Focal spot } \\
\text { (Actual) }\end{array}$ \\
\hline 1 & 0.6 & 6.67 & 2.0 & $2.6 \times 3.64$ \\
\hline 2 & 0.7 & 5.71 & 2.0 & $2.6 \times 3.64$ \\
\hline 3 & 0.85 & 4.71 & 2.0 & $2.6 \times 3.64$ \\
\hline 4 & 1.0 & 4.00 & 2.0 & $2.6 \times 3.64$ \\
\hline 5 & 1.15 & 3.48 & 1.8 & $2.34 \times 3.28$ \\
\hline 6 & 1.4 & 2.86 & 1.5 & $1.95 \times 2.73$ \\
\hline 7 & 1.7 & 2.35 & 1.3 & $1.5 \times 2.18$ \\
\hline 8 & 2.0 & 2.00 & 1.0 & $1.4 \times 1.96$ \\
\hline 9 & 2.5 & 1.60 & 0.8 & $1.12 \times 1.57$ \\
\hline 10 & 2.8 & 1.43 & 0.6 & $0.90 \times 1.26$ \\
\hline
\end{tabular}

The wire-mesh test tool is placed on the top of the cassette. In the processed radiograph, areas of poor screen-film contact appear as dark areas (which show the blurred images of the wire) and good screen-film contact (evident by uniform density throughout the radiograph). A radiograph of the film screen contact test is shown in Fig. 3.

\subsection{Half Value Layer (HVL)}

In the present study, the half-value layer (HVL) which is a measure of quality of the x-ray beam is determined by using a Gammex RMI Step Wedge. For this measurement, the loaded cassette is placed on the couch at a distance $100 \mathrm{~cm}$ from the tube-target. The step-wedge is placed over the cassette, and the beam is collimated over the step-wedge. A resulting radiograph of the HVL test is shown in Fig. 4. Optical densities of the processed film are determined for

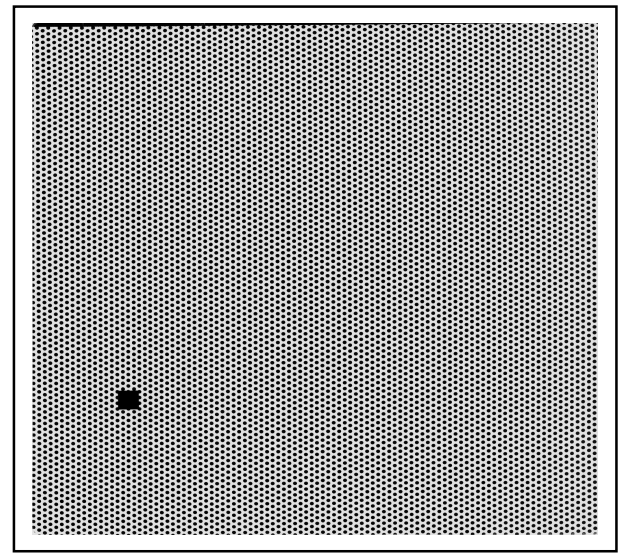

Fig.3: Radiograph of film screen contact test 
different thickness of the step-wedge with the help of a densitometer which measures the degree of darkness (optical density) of a photographic film.

The densitometer is calibrated with calibration film of 2.38 optical densities. Before having each measurement by using the densitometer, it is mandatory to make a zero-processing. The measurement of the measuring field is performed by pushing down the measuring arm until the

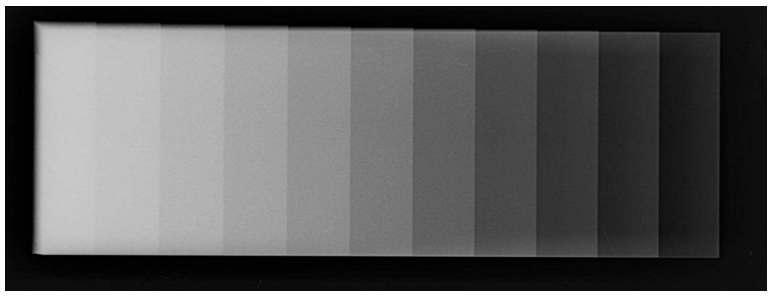

Fig.4: Radiograph of half value layer (HVL) test measuring value is displayed. The densitometer switches off automatically after a period of 90 seconds without impulse and then can be reactivated by newly pushing the measuring arm. The measuring range of the densitometer is $\mathrm{O} \leq \mathrm{D} \leq 4$ and the values are plotted for "thickness (mm)" versus "optical densities". From the graphs, the HVL of x-ray beams are determined for 40 different diagnostic x-ray facilities. The requirement [12] for the minimum HVL for the x-ray beams are indicated in Table 2.

\section{RESULTS AND DISCUSSION}

Some important quality control tests in forty (40) diagnostic x-ray facilities which are performed to obtain high quality diagnostic images are shown in Table 3 .

\subsection{Congruence between Optical and Radiation Field}

In the present study, $\mathrm{kV}_{\mathrm{p}}$ is found to vary from 50 $\mathrm{kV}_{\mathrm{p}}$ to $70 \mathrm{kV}_{\mathrm{p}}$ and $\mathrm{mA}$ from $30 \mathrm{~mA}$ to $320 \mathrm{~mA}$ with a film-to-focus distance (FFD) maintained fixed at $100 \mathrm{~cm}$ (with maximum allowed misalignment of 2\%). Fig. 5(a) shows the number of machines for which congruence within limit and out of limit and the graph shows that $77.5 \%$ facilities results are within the limits, on the other hand, the remaining $(22.5 \%)$ facilities fall outside the limits, reducing diagnostic image quality and leading to exposure of non-targeted areas. Misalignment may be caused due to shifts in the relative positions of the light bulb, reflecting mirror or anode focal spot. With appropriate precautions, radiation doses can be kept within the recommended limits.
Table 2: Minimum HVL requirement for an $\mathrm{X}$-ray unit

\begin{tabular}{|c|c|c|}
\hline $\begin{array}{c}\text { Designed } \\
\text { operating } \\
\text { potential } \\
(\mathrm{kVp})\end{array}$ & $\begin{array}{c}\text { Measured } \\
\text { operating } \\
\text { potential } \\
(\mathrm{kVp})\end{array}$ & $\begin{array}{c}\text { Minimum } \\
\mathrm{HVL} \\
(\mathrm{mm} \text { of } \mathrm{Al})\end{array}$ \\
\hline \multirow{3}{*}{$<51$} & 30 & 0.3 \\
\cline { 2 - 3 } & 40 & 0.4 \\
\hline \multirow{3}{*}{$51-70$} & 50 & 0.5 \\
\cline { 2 - 3 } & 51 & 1.2 \\
\cline { 2 - 3 } & 60 & 1.3 \\
\hline \multirow{5}{*}{$>70$} & 70 & 1.5 \\
\cline { 2 - 3 } & 71 & 2.1 \\
\cline { 2 - 3 } & 80 & 2.3 \\
\cline { 2 - 3 } & 90 & 2.5 \\
\cline { 2 - 3 } & 100 & 2.7 \\
\cline { 2 - 3 } & 110 & 3.0 \\
\cline { 2 - 3 } & 120 & 3.2 \\
\cline { 2 - 3 } & 130 & 3.5 \\
\cline { 2 - 3 } & 140 & 3.8 \\
\hline
\end{tabular}

\subsection{Beam Alignment}

Beam alignment is checked at different diagnostic $x$-ray facilities to determine the perpendicularity of the $\mathrm{x}$-ray beam to the image receptor. In the present investigation, the tolerance limit is defined to be $1.5^{0}$ from the perpendicular for an FFD of $100 \mathrm{~cm}$. The $\mathrm{kV}_{\mathrm{p}}$ varied from $50 \mathrm{kV}_{\mathrm{p}}$ to $70 \mathrm{kV}_{\mathrm{p}}$, and the $\mathrm{mA}$ from $30 \mathrm{~mA}$ to $320 \mathrm{~mA}$. The results from the investigation are given in Fig. 5 (b), which indicates that $60 \%$ of the facilities are within the limit [12]. The facilities which fell outside the limit should manually adjust their x-ray beam to make it perpendicular to the image receptor in order to reduce radiographic image distortion 
Table 3: Quality control tests for different parameters of diagnostic X-ray installations

\begin{tabular}{|c|c|c|c|c|c|c|c|}
\hline $\begin{array}{l}\text { Serial } \\
\text { no. }\end{array}$ & $\begin{array}{l}\text { Machine } \\
\text { code }\end{array}$ & $\begin{array}{l}\text { Congruence } \\
\text { between optical } \\
\text { and radiation field } \\
(*)\end{array}$ & $\begin{array}{l}\text { Beam } \\
\text { alignment } \\
(* *)\end{array}$ & $\begin{array}{l}\text { Resolved } \\
\text { group }\end{array}$ & $\begin{array}{l}\text { Focal spot } \\
\text { size } \\
\text { (Nominal) } \\
(\mathrm{mm})\end{array}$ & $\begin{array}{l}\text { Film-screen } \\
\text { contact }\end{array}$ & $\begin{array}{l}\text { HVL } \\
(\mathrm{mm})\end{array}$ \\
\hline 1. & $\mathrm{X}-1$ & Within Limit & Within Limit & 7 & 1.3 & Uniform & 17 \\
\hline 2. & $\mathrm{X}-2$ & Within Limit & Within Limit & 5 & 1.8 & Uniform & 16 \\
\hline 3. & $\mathrm{X}-3$ & Out of Limit & Within Limit & 5 & 1.8 & Uniform & 12 \\
\hline 4. & $\mathrm{X}-4$ & Within Limit & Within Limit & 1 & 2.0 & Uniform & 19 \\
\hline 5. & $\mathrm{X}-5$ & Within Limit & Out of Limit & 1 & 2.0 & Uniform & 29 \\
\hline 6. & $\mathrm{X}-6$ & Within Limit & Within Limit & 1 & 2.0 & Non Uniform & 22 \\
\hline 7. & $\mathrm{X}-7$ & Within Limit & Within Limit & 4 & 2.0 & Non Uniform & 25 \\
\hline 8. & $\mathrm{X}-8$ & Within Limit & Out of Limit & 5 & 1.8 & Uniform & 16 \\
\hline 9. & $\mathrm{X}-9$ & Within Limit & Out of Limit & 5 & 1.8 & Non Uniform & 21 \\
\hline 10. & $\mathrm{X}-10$ & Within Limit & Within Limit & 7 & 1.3 & Uniform & 11 \\
\hline 11. & $\mathrm{X}-11$ & Within Limit & Out of Limit & 1 & 2.0 & Non Uniform & 12 \\
\hline 12. & $\mathrm{X}-12$ & Within Limit & Out of Limit & 3 & 2.0 & Non Uniform & 24 \\
\hline 13. & $\mathrm{X}-13$ & Within Limit & Out of Limit & 1 & 2.0 & Non Uniform & 18 \\
\hline 14. & $\mathrm{X}-14$ & Within Limit & Within Limit & 1 & 2.0 & Uniform & 17 \\
\hline 15. & $\mathrm{X}-15$ & Within Limit & Out of Limit & 7 & 1.3 & Uniform & 22 \\
\hline 16. & $\mathrm{X}-16$ & Within Limit & Out of Limit & 2 & 2.0 & Uniform & 10 \\
\hline 17. & $\mathrm{X}-17$ & Out of Limit & Within Limit & 2 & 2.0 & Uniform & 21 \\
\hline 18. & $\mathrm{X}-18$ & Within Limit & Within Limit & 4 & 2.0 & Uniform & 20 \\
\hline 19. & X-19 & Out of Limit & Within Limit & 6 & 1.5 & Uniform & 12 \\
\hline 20. & $\mathrm{X}-20$ & Within Limit & Out of Limit & 2 & 2.0 & Uniform & 7 \\
\hline 21. & $\mathrm{X}-21$ & Within Limit & Out of Limit & 2 & 2.0 & Uniform & 13 \\
\hline 22. & $X-22$ & Out of Limit & Within Limit & 1 & 2.0 & Non Uniform & 21 \\
\hline 23. & $\mathrm{X}-23$ & Within Limit & Within Limit & 4 & 2.0 & Uniform & 10 \\
\hline 24. & $\mathrm{X}-24$ & Within Limit & Within Limit & 1 & 2.0 & Uniform & 06 \\
\hline 25. & $X-25$ & Out of Limit & Out of Limit & 7 & 1.3 & Uniform & 26 \\
\hline 26. & $\mathrm{X}-26$ & Within Limit & Within Limit & 5 & 1.8 & Uniform & 14 \\
\hline 27. & $\mathrm{X}-27$ & Within Limit & Out of Limit & 1 & 2.0 & Non Uniform & 24 \\
\hline 28. & $\mathrm{X}-28$ & Within Limit & Within Limit & 1 & 2.0 & Non Uniform & 20 \\
\hline 29. & $\mathrm{X}-29$ & Within Limit & Within Limit & 1 & 2.0 & Uniform & 26 \\
\hline 30. & $\mathrm{X}-30$ & Out of Limit & Out of Limit & 4 & 2.0 & Uniform & 22 \\
\hline 31. & $\mathrm{X}-31$ & Out of Limit & Out of Limit & 4 & 2.0 & Non Uniform & 17 \\
\hline 32. & $\mathrm{X}-32$ & Within Limit & Within Limit & 4 & 2.0 & Uniform & 08 \\
\hline 33. & $\mathrm{X}-33$ & Within Limit & Within Limit & 6 & 1.5 & Uniform & 14 \\
\hline 34. & $\mathrm{X}-34$ & Out of Limit & Within Limit & 4 & 2.0 & Non Uniform & 18 \\
\hline 35. & $\mathrm{X}-35$ & Within Limit & Out of Limit & 3 & 2.0 & Uniform & 11 \\
\hline 36. & $\mathrm{X}-36$ & Within Limit & Out of Limit & 1 & 2.0 & Uniform & 31 \\
\hline 37. & $\mathrm{X}-37$ & Out of Limit & Within Limit & 4 & 2.0 & Non Uniform & 13 \\
\hline 38. & $\mathrm{X}-38$ & Within Limit & Within Limit & 6 & 1.5 & Uniform & 12 \\
\hline 39. & X-39 & Within Limit & Within Limit & 3 & 2.0 & Non Uniform & 26 \\
\hline 40. & $\mathrm{X}-40$ & Within Limit & Within Limit & 1 & 2.0 & Non Uniform & 13 \\
\hline
\end{tabular}

(*) Tolerance Limit: Maximum misalignment allowed is $2 \%$ of film-focus distance.

(**) Tolerance Limit: $1.5^{0}$ from perpendicular which is applicable to film-to-focus distance of $40^{\prime \prime}$. 


\subsection{Focal Spot Size}

A bar pattern focal spot test tool is used in this work to determine the nominal focal spot size of the diagnostic $\mathrm{x}$-ray equipment. The $\mathrm{kV}_{\mathrm{p}}$ varies from $45 \mathrm{kV}_{\mathrm{p}}$ to $70 \mathrm{kV}_{\mathrm{p}}$ and the $\mathrm{mA}$ from $30 \mathrm{~mA}$ to $320 \mathrm{~mA}$. Fig. 5 (c) shows that, $92.5 \%$ of the time, the focal spot size of diagnostic x-ray machines are matched perfectly with the x-ray tube's indicated rating of focal spot size; the remaining are altered due to bombardment of electrons on the targets. To rectify focal spot size condition, the x-ray facilities should evaluate it periodically as size and focal spot shape tend to deteriorate with use; users should also refer to the focal spot size rating written on the x-ray tube.

\subsection{Film-Screen Contact}

Film-Screen contact are checked at different diagnostic x-ray facilities in order to assess any blur or dark patches in the image as a result of poor contact between film and screen. The values obtained from the measurement are shown in Table 3. In this investigation, $\mathrm{kV}_{\mathrm{p}}$ varies from $48 \mathrm{kV}_{\mathrm{p}}$ to $70 \mathrm{kV}_{\mathrm{p}}$ and $\mathrm{mA}$ from $30 \mathrm{~mA}$ to $320 \mathrm{~mA}$; the FFD is $100 \mathrm{~cm}$. Fig. 5 (d) shows the information regarding the film-screen contact of the present study. From the data, it is found that $65 \%$ of the facilities are uniform. Most of the facilities provide good film-screen contact with evenly spread density. On the other hand, 35\% of the facilities produce poor quality diagnostic images that lead to improper diagnosis. Therefore, to obtain proper information about treatment from radiographic images, these facilities must improve the situation by minimizing the poor contact between film and screen, which may be caused by a defective cassette or film changer that does not apply sufficient pressure over the entire film area and gives rise to diffusion of light emitted by the intensifying screen leading to image blurring.

\subsection{Half-Value Layer (HVL)}

The half-value layer (HVL) of the x-ray beam is determined by plotting optical density vs. thickness $(\mathrm{mm})$ for different diagnostic x-ray films which are exposed with a Gammex RMI step wedge. In this case, the $\mathrm{kV}_{\mathrm{p}}$ is varied from $50 \mathrm{kV}_{\mathrm{p}}$ to $75 \mathrm{kV}_{\mathrm{p}}$ and the $\mathrm{mA}$ from $30 \mathrm{~mA}$ to $320 \mathrm{~mA}$. The values obtained from this part of the study are listed in Table 3. A typical half-value layer measurement is shown in fig.5 (e). The observed magnitudes of the HVLs vary between $6 \mathrm{~mm}$ and $31 \mathrm{~mm}$, far above the recommended values (e. g., $2.1 \mathrm{~mm}$ ) [12]. Values so high in magnitude could not be explained, although some variation is expected as HVL is a function of $\mathrm{x}$-ray tube age (use), voltage waveform and kilo peak voltage ( $\mathrm{kVp}$ ). Facilities should measure HVL at least annually or after replacement of the x-ray tube assembly, and should also use adequate filtration to provide improved quality diagnostic images with radiation safety.

\section{CONCLUSIONS}

The main focus of the present study is to investigate the important parameters of the quality control programme for the x-ray machines under investigation and, consequently, their influence on radiation exposure to patients toward the effective and safe use of $x$ rays. Quality control is an integral and the most tangible aspect of quality assurance, and involves the selective testing of each major system component on a regular basis. Significant reduction of the population dose can be achieved through the control and reduction of the doses to patients undergoing $\mathrm{x}$-ray examinations. Certain actions should be taken to ensure the safe and proper operation of the x-ray system, improving equipment performance and image quality and reducing patient doses. Quality control on a regular basis as well as routine servicing may lead to a substantial reduction in variations from the normal performance and system malfunctioning. During a radiological examination, irradiation of the patient should be minimized by using the best available techniques, and measures should be taken to reduce, as far as possible, the dose to other parts of the body consistent with the clinical needs of each case. Further, the following guidelines are suggested to maintain proper quality control in diagnostic x-ray facilities: 
- $\quad$ Congruence between optical and radiation field must be within the limit of $2 \%$ of the film-focus distance (FFD) as per quality control protocol to obtain improved quality diagnostic images.

- Diagnostic x-ray beam should be perpendicular to the image receptor, which prevents radiographic image distortion.

- Focal spot size should be checked periodically to ensure that it is not altered as a result of bombardment of electrons on the target.

- $\quad$ Radiographic film must be firmly contacted with the intensifying screen to reduce the chance of improper diagnosis.

- $\quad$ Half-value layer (HVL) of the x-ray beam should be determined to judge the beam quality, which may make it possible to minimize unwanted radiation exposure and the need for retakes.

On the basis of this work, it is possible to provide a guideline on the sort of allowable variations in performance of the x-ray facilities, and to support corrective actions to avoid occurrences of poor quality diagnostic images. Ultimately, this approach will lead to a reduction of radiation hazards in the field. The primary goal of the study is to establish the baseline quality control parameters for different $\mathrm{x}$-ray facilities and to ensure good quality images which lead to get better information during image analysis.

\section{REFERENCES}

1. Ajayi IR, Akinwumiju A. Measurement of entrance surface dose to patients in four common diagnostic examinations by thermoluminescence dosimetry in Nigeria. Radiation Protection Dosimetry 2000;87:217-20.

2. Williams JR, Fipem, Catting MK. An investigation of X-ray equipment factors influencing patient dose in radiography. The British Journal of Radiology 1998;71:1192-98.

3. Almen A, Tingberg A, Mattsson S, Besjakov J, Kheddache S, Lanhede B, Mansson LG, Zankl M. The influence of different technique factors on image quality of lumbar spine radiographs as evaluated by established CEC image criteria. The British Journal of Radiology 2000;73:1192-99.

4. Hourdakis CJ, Papageorgiou E, Tritakis P, Manousaridis G, Hadjiantoniou A. A national survey: Performance of medical radiographic X-ray systems in Greece. Radiation Protection Dosimetry 1999;81:195-203.

5. Muhogora WE, Nyanda AM. The potential for reduction of radiation doses to patients undergoing some common X-ray examinations in Tanzania. Radiation Protection Dosimetry 2001;94:381-84.

6. Freitas MB, Yoshimura EM. An overview of doses to patients and irradiation conditions of diagnostic chest Xray examinations carried out in hospitals of the city of Sao Paulo, Brazil. Radiation Protection Dosimetry 2003;103:141-48.

7. Hamed AA, Elshirbiny N, Nassef M. Study of radiation exposure dependence on the physical parameters of medical diagnostic X-ray machines. Radiation Protection Dosimetry 1999;82:277-83.

8. International Commission on Radiological Protection. Recommendations of the International Commission on Radiological Protection, Publication 60. Oxford: Pergamon Press, 1990.

9. Warren-forward HM, McKeeney DB. Towards reduction of patient exposure in medical diagnostic radiology. Radiation Protection Dosimetry 1992;43:283-86.

10. Jankowski J, Staniszewska. Methodology for the set-up of a quality control system for diagnostic x-ray units in Poland.Radiation Protection Dosimetry 2000;90:259-62.

11. Schandorf C, Tetteh GK. Analysis of the status of X-ray diagnosis in Ghana. The British Journal of Radiology 1998;71:1040-48.

12. Rehani M M, Diagnostic Imaging Quality Assurance, $1^{\text {st }}$ edition. Jaypee Brothers Medical Publishers (P) Ltd., 1995.

13. AAPM Report no.74, Report of Task Group-12. Diagnostic x-ray imaging committee, Quality control in diagnostic radiology, United States of America, Medical physics publishing, International standard book number: 1-888340-33-9.Available from:http://www medicalphysics.org, July 2002. 
14. Conference of Radiation Control Program Directors, Ins, Center for Devices and Radiological Health Food and Drug Administrations, Quality Control Recommendations for Diagnostic Radiography, vol.3 Radiographic or Fluoroscopic Machines. published by conference of Radiation control program Directors, Ins, July 2001. Available from:http://www.crcpd.org.

15. New York State. Department of Health Guide for Radiation Safety/Quality Assurance Programs, Revised: May 2007. 